\title{
Electroweak structure of light nuclei within chiral effective field theory
}

\author{
Received: date / Accepted: date
}

\begin{abstract}
We review the results of the most recent calculations for the electromagnetic structure of light nuclei, the weak muon capture on deuteron and ${ }^{3} \mathrm{He}$ and the weak proton-proton capture reaction at energies of astrophysical interest, performed within the chiral effective field theory framework.
\end{abstract}

Keywords Chiral effective field theory · Electromagnetic Form Factors · Light nuclei · Muon capture · Proton-proton weak capture

\section{Introduction}

Among the great advantages of the chiral effective field theory $(\chi \mathrm{EFT})$ framework, the two following ones are here of interest: (i) the possibility of deriving nuclear electroweak (EW) currents consistently with the nuclear interaction, and (ii) the possibility of setting a hierarchy among the different contributions, both for the interactions and the currents. In fact, it is well known that $\chi \mathrm{EFT}$ can justify $a$ priori the empirical observation that the contribution of three-nucleon interactions to nuclear structure is far less significant than that of the two-nucleon force. Furthermore, the $\chi$ EFT power counting allows to recognize which are the most significant contributions also among the different currents.

The idea of using $\chi$ EFT to derive the nuclear EW transition operators was first implemented by Park et al. [1] in the nineties. They derived the nuclear electromagnetic (EM) current and charge operators, within the so-called heavy-baryon chiral perturbation theory $(\mathrm{HB} \chi \mathrm{PT})$ approach, where the baryons are treated as heavy static sources, and the perturbative expansion is performed in terms of the involved momenta over the baryon mass. The weak axial current and charge operators were derived by the same authors few years later [2], and applied to weak reactions of astrophysical interest within a "hybrid" approach, in which nuclear wave functions were obtained from phenomenological potentialsthe $\chi \mathrm{EFT}$ potentials available at the time were not yet as accurate as the phenomenological ones. Only very recently, these $\chi \mathrm{EFT}$ weak operators have been used to study weak processes which involve fewnucleon systems in conjunction with nowadays accurate $\chi$ EFT potentials, in particular the two-nucleon (NN) potential derived at next-to-next-to-next-to leading order (N3LO) by Entem and Machleidt 3], augmented, when needed, by the three-nucleon interaction (TNI) obtained at next-to-next-to leading order (N2LO), in the version of Ref. [4]. In particular, the muon captures on deuteron and ${ }^{3} \mathrm{He}$, in the non-breakup channel [5], and the proton-proton weak capture (the so-called $p p$ reaction), in a wide energy range [6] have been considered.

Laura E. Marcucci

University of Pisa and INFN-Pisa, I-56127 Pisa (Italy) and Gran Sasso Science Institute (INFN), I-67100 L'Aquila (Italy)

Tel.: $+39-050-2214901$

Fax: +39-050-2214887

E-mail: laura.marcucci@df.unipi.it 


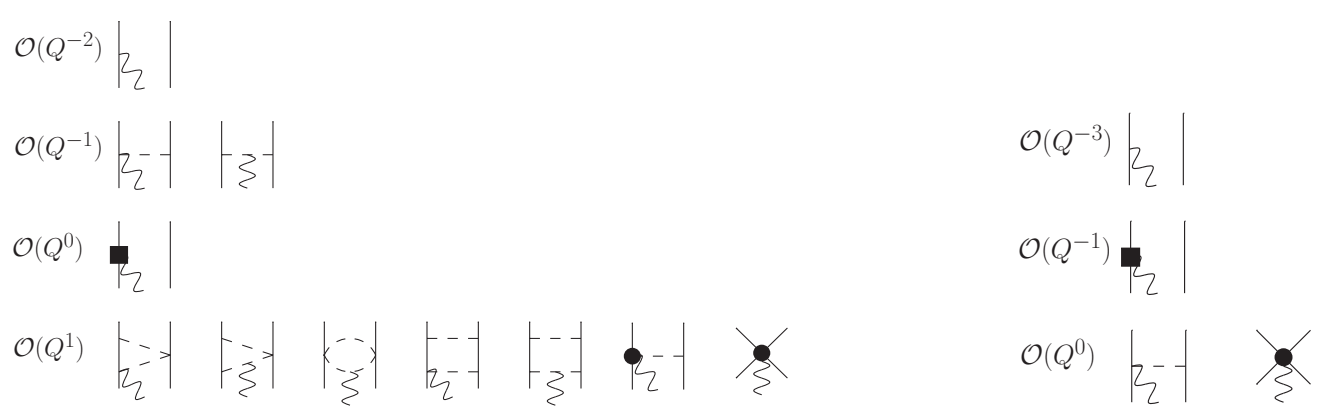

Fig. 1 On the left: diagrams illustrating one- and two-body $\chi$ EFT EM currents entering at LO $\left(Q^{-2}\right)$, NLO $\left(Q^{-1}\right)$, N2LO $\left(Q^{0}\right)$, and N3LO $\left(Q^{1}\right)$. On the right: diagrams illustrating one- and two-body $\chi$ EFT axial currents entering at LO $\left(Q^{-3}\right)$, NLO $\left(Q^{-1}\right)$, and N2LO $\left(Q^{0}\right)$. Nucleons, pions, and EW probes are denoted by solid, dashed, and wavy lines, respectively. The solid square represents the relativistic corrections to the one-body current, while the solid circles represent the contact terms. Only the relevant topologies are indicated. Loop corrections to short range EM currents turn out to vanish. No contribution of order $Q^{-2}$ exists for the axial current.

Few years ago, the problem of deriving the EM current and charge operators in $\chi$ EFT has been revisited by Pastore et al. [7] and, in parallel, by Kölling et al. [8]. Pastore et al. have used timeordered perturbation theory (TOPT) to calculate the EM transition amplitudes, which allows for an easier treatment of the so-called reducible diagrams than the $\mathrm{HB} \chi \mathrm{PT}$ approach. On the other hand, Kölling et al. have used the method of unitary transformation, the same one used to derive the chiral potentials mentioned above. We will focus here only on the work of Pastore et al., but we would like to remark that the results obtained by these two groups, although with different methods, are in good agreement with each other. The new set of NN EM currents derived by Pastore et al. have been found significantly different from those of Park et al., as it will be discussed in the next section, where the $\chi$ EFT EW operators will be reviewed. This contribution then continues with Sec. 3, where we present the results of the most recent $\chi \mathrm{EFT}$ calculations of EW observables, in particular the EM structure of $A=2,3$ nuclei [9], the rate for muon captures on deuteron and ${ }^{3} \mathrm{He}$ [5], and the $p p$ reaction astrophysical $S$-factor [6]. Some concluding remarks will be presented in Sec. 4.

\section{Electroweak transition operators}

The EW transition operators consist of six terms: the EM and the weak axial and vector charge and current operators. The weak vector current and charge operators can be related to the corresponding EM ones applying the conserved-vector-current (CVC) hypothesis, which basically links these via a rotation in the isospin space. Therefore, we choose to consider the EM and axial operators. In this contribution, we will limit ourselves to the current operators, due to limitation of space. Both $\chi \mathrm{EFT}$ current operators can be expanded in powers of pions' and nucleons' momenta, $Q$, and consist of long- and intermediate-range components which are described in terms of one- and two-pion exchange contributions, as well as contact currents which encode the short-range physics. These last operators involve a number of so-called low-energy constants (LECs), to be fixed to experimental data.

Electromagnetic current operator. The EM current operators are diagrammatically represented in the left panel of Fig. 1, where they are listed according to their scaling in $Q$. The leading order (LO) contribution consists of the well known single-nucleon convection and magnetization currents, and is of order $Q^{-2}$, while the next-to-next-to leading order (N2LO) contribution arises from the $(Q / m)^{2}$ relativistic corrections to the previous contribution ( $m$ is the nucleon mass), and is therefore of order $Q^{0}$. The next-to-leading order (NLO) term involves seagull and in-flight long-range contributions associated with one-pion exchange (OPE). The next-to-next-to-next-to-leading order (N3LO) currents, therefore at order $Q^{1}$, consist of (i) one-loop two-pion-exchange (TPE) terms, (ii) OPE terms induced by $\gamma \pi N$ interactions beyond LO, and (iii) contact terms generated by minimal substitution in the four-nucleon contact interactions involving two gradients of the nucleon fields, as well as by nonminimal couplings to the electromagnetic field. The former are linked to the $\chi$ EFT potential at order $Q^{2}$ via current 
conservation, and therefore they involve the same LECs entering the $\chi \mathrm{EFT} \mathrm{NN}$ interaction. These are taken from fits to the NN scattering data. On the other hand, the LECs entering the nonminimal contact currents as well as those entering the N3LO OPE contribution need to be fixed to EM observables. The explicit expression for all these N3LO currents can be found in Ref. [9]. Note that the two-body $\chi$ EFT operators have a power-law behavior at large momenta, which requires a regularization procedure. This is implemented via the introduction of a cutoff function of the form $\exp \left(-Q^{4} / \Lambda^{4}\right)$, where $\Lambda=500$ or $600 \mathrm{MeV}$ (the same values of the NN potential and TNI).

We consider in some detail only those N3LO contributions involving new LECs, i.e., the nonminimal and OPE currents. These terms can be written as

$$
\begin{aligned}
\mathbf{J}_{\mathrm{nm}}(i j) & \propto \mathbf{q} \times\left[d_{1}^{S} \boldsymbol{\sigma}_{i}+d_{1}^{V}\left(\tau_{i}^{z}-\tau_{j}^{z}\right) \boldsymbol{\sigma}_{i}\right]+i \rightleftharpoons j \\
\mathbf{J}_{\mathrm{OPE}}(i j) & \propto \frac{\boldsymbol{\sigma}_{j} \cdot \mathbf{k}_{j}}{\left(m_{\pi}^{2}+\mathbf{k}_{j}^{2}\right)} \mathbf{q} \times\left[\left(d_{2}^{S} \boldsymbol{\tau}_{i} \cdot \boldsymbol{\tau}_{j}+d_{2}^{V} \tau_{j}^{z}\right) \mathbf{k}_{j}+d_{3}^{V}\left(\boldsymbol{\tau}_{i} \times \boldsymbol{\tau}_{j}\right)^{z} \boldsymbol{\sigma}_{i} \times \mathbf{k}_{j}\right]+i \rightleftharpoons j,
\end{aligned}
$$

where $\mathbf{q}$ is the photon momentum, $\boldsymbol{\sigma}_{i}\left(\boldsymbol{\tau}_{i}\right)$ are the spin (isospin) Pauli matrices, $\mathbf{k}_{i}$ is the momentum transfer to nucleon $i$. The LECs $d_{1}^{S}, d_{1}^{V}, d_{2}^{S}, d_{2}^{V}$ and $d_{3}^{V}$ need to be fixed on EM observables. The adopted fitting procedure is extensively discussed in Ref. [9]. Here we only summarize the main features: (i) the LECs multiplying isoscalar operators $\left(d_{1}^{S}\right.$ and $\left.d_{2}^{S}\right)$ are fixed so as to reproduce the deuteron magnetic moment and the isoscalar combination of the $A=3$ magnetic moments $\left(\mu_{S}\right)$. (ii) In order to achieve "natural" values for the LECs multiplying isovector operators and not to spoil chiral convergence, two LECs $\left(d_{2}^{V}\right.$ and $\left.d_{3}^{V}\right)$ have been fixed by saturating the $\Delta$-resonance (a common strategy adopted in the literature), and $d_{1}^{V}$ has been fixed by fitting either the cross section for neutron-proton radiative capture at thermal energies, $\sigma_{n p}$, or the isovector combination of the $A=3$ magnetic moments, $\mu_{V}$. These two sets of LECs are called SET II and SET III, respectively. The values for the different LECs are given in Ref. [9], where it has been shown that the experimental value for $\sigma_{n p}\left(\mu_{V}\right)$ is reproduced within few percent with the LECs of SET III (SET II).

Finally, we would like to remark that the most significant differences between the model for the EM current presented here and that of Park et al. arise at N3LO for the box diagrams and the contact terms. In particular, the contact terms of Park et al. are much simpler than those presented above and can be written as sum of two terms, one isoscalar and one isovector, with two different LECs in front. These LECs have been fitted in Refs. [5; 6] to reproduce $\mu_{S}$ and $\mu_{V}$. For a more detailed discussion of this point, see Ref. [7].

Weak axial current operator. The weak axial current operators are diagrammatically represented in the right panel of Fig. 1, where, as in the EM case, they are listed according to their scaling in $Q$. The LO contribution consists of the well known single-nucleon axial current, and is of order $Q^{-3}$. At order $Q^{-2}$ it turns out that there are no contributions, and therefore the NLO contribution is of order $Q^{1}$, and arises from the $(Q / m)^{2}$ relativistic corrections to the LO contribution. The N2LO currents consist of the OPE term and a contact term. Note that N3LO contributions arise from loop and TPE terms, and they have not been calculated yet. They are not illustrated in Fig. 1. The only model available for the axial current is that of Park et al. 2], up to N2LO, with only one LEC, $d_{R}$. As first shown in Ref. [10], such LEC can be related to the LEC $c_{D}$ entering one of the two contact terms present in the TNI at N2LO, via the relation $d_{R}=\frac{m}{\Lambda_{\chi} g_{A}} c_{D}+\frac{1}{3} m\left(c_{3}+2 c_{4}\right)+\frac{1}{6}$, where $g_{A}$ is the single-nucleon axial coupling constant, $c_{3}$ and $c_{4}$ are LECs of the $\pi N$ Lagrangian, already part of the chiral NN potential at $\mathrm{NLO}$, and $\Lambda_{\chi}=700 \mathrm{MeV}$ is the the chiral-symmetry-breaking scale. Therefore, it has become common practice to fit $c_{D}$ (and $c_{E}$ - the other LEC entering the N2LO TNI) to the triton binding energy and the Gamow-Teller matrix element in tritium $\beta$-decay. The values obtained in this way for $c_{D}$ and $c_{E}$ are listed in Ref. [5] and they have been used in Refs. [5; 6] to study the muon capture on deuteron and ${ }^{3} \mathrm{He}$ and the $p p$ capture, as it will be reviewed in Sec. 3. Note that the first studies of $A=3$ and 4 elastic scattering observables, as cross sections and analyzing powers, with these values for $c_{E}$ and $c_{D}$ have been reported in Ref. 11]. A final remark: the model of Park et al. is the only one available at present for both the axial and the vector (or EM) current operators. We have used this model in our studies of weak observables [5; 6]. However, the significant differences found between the Park et al. model and the most recent ones of Pastore et al. (and Kölling et al.) in the EM sector make it urgent to perform a new derivation of the weak axial operators within TOPT, following the footsteps of Pastore et al. for the EM case. 
Table 1 Deuteron r.m.s. radius $\left(r_{d}\right)$ and quadrupole moment $\left(Q_{d}\right)$, and ${ }^{3} \mathrm{H}$ and ${ }^{3} \mathrm{He}$ charge $\left(r_{c}\right)$ and magnetic $\left(r_{m}\right)$ radii. The corresponding experimental values are also reported.

\begin{tabular}{lll}
\hline & Theory & Experiment \\
\hline$r_{d}[\mathrm{fm}]$ & $1.972 \pm 0.004$ & $1.9733 \pm 0.0044$ \\
$Q_{d}\left[\mathrm{fm}^{2}\right]$ & $0.2836 \pm 0.0016$ & $0.2859 \pm 0.0003$ \\
$r_{c}\left({ }^{3} \mathrm{He}\right)[\mathrm{fm}]$ & $1.962 \pm 0.004$ & $1.959 \pm 0.030$ \\
$r_{c}\left({ }^{3} \mathrm{H}\right)[\mathrm{fm}]$ & $1.756 \pm 0.006$ & $1.755 \pm 0.086$ \\
$r_{m}\left({ }^{3} \mathrm{He}\right)[\mathrm{fm}]$ & $1.905 \pm 0.022$ & $1.965 \pm 0.153$ \\
$r_{m}\left({ }^{3} \mathrm{H}\right)[\mathrm{fm}]$ & $1.791 \pm 0.018$ & $1.840 \pm 0.181$ \\
\hline
\end{tabular}
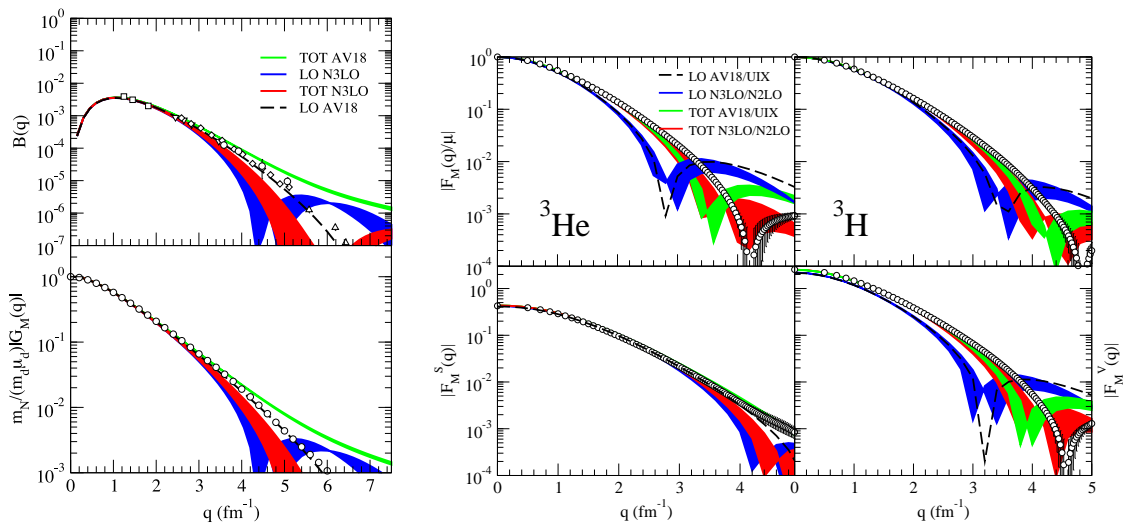

Fig. 2 The deuteron $B(q)$ structure function and magnetic form factor (on the left), and the $A=3$ magnetic form factors, together with their isoscalar and isovector combinations (on the right), obtained at leading order (LO) and with inclusion of current operators up to N3LO (TOT), compared with the experimental data. The curves labelled "AV18" (or "AV18/UIX") have been obtained within the hybrid $\chi$ EFT approach using the AV18 (and Urbana IX 13 TNI for $A=3$ ) phenomenological potentials. The curves labelled "N3LO" (or "N3LO/N2LO") have been obtained instead using the N3LO NN potential [3] (and N2LO [4] TNI for $A=3$ ).

\section{Results}

In this section we first present some selected results on the electromagnetic structure of the deuteron, the triton and ${ }^{3} \mathrm{He}$. A complete discussion can be found in Ref. 9]. Then we present results for the muon capture on deuteron and ${ }^{3} \mathrm{He}$ [5], and for the $p p$ reaction [6], respectively.

The electromagnetic structure of $A=2,3$ nuclei. The static properties of $A=2,3$ nuclei are summarized in Table 1, where we present the $\chi$ EFT results for the deuteron r.m.s. radius and quadrupole moment, and the charge and magnetic radii for the $A=3$ nuclei. The experimental data are also reported. Note that the deuteron and $A=3$ magnetic moments are used to fit the LECs (in SET III). The theoretical uncertainties are due to the cutoff dependence and the fitting procedure. By inspection of the table, we can conclude that the static properties of the $A=2,3$ nuclei are nicely reproduced. It should be noticed that within the phenomenological approach, based on the Argonne $v_{18}$ potential 12 ] (AV18), the quadrupole moment is calculated to be $0.275 \mathrm{fm}^{2}$, in significant disagreement with the experimental value.

The deuteron $B(q)$ structure function and magnetic form factor, together with the $A=3$ magnetic form factors, and their isoscalar and isovector combinations, are given in Fig. 2. By inspection of the figure, we can conclude that the $\chi \mathrm{EFT}$ calculation is in agreement with the experimental data in a range of $q$-values much larger than one would naively expect, (up to 1-2 times the pion mass). On the other hand, this $\chi \mathrm{EFT}$ calculation is unable to reproduce the first diffraction region of the $A=3$ magnetic form factors, a problem which is present also in the hybrid calculation, as well as in the phenomenological one (see for instance Ref. 14]). 
Table 2 Doublet capture rate for muon capture on deuteron and total capture rate for muon capture on ${ }^{3}$ He obtained at leading order (LO) and with inclusion of current operators up to N2LO (TOT). The results calculated with the different values of two cutoff $\Lambda$ are reported. The theoretical uncertainties are due to the fitting procedure of the LEC $d_{R}$.

\begin{tabular}{llll}
\hline & $\Gamma^{D}\left({ }^{1} S_{0}\right)\left[\mathrm{sec}^{-1}\right]$ & $\Gamma^{D}\left[\mathrm{sec}^{-1}\right]$ & $\Gamma_{0}\left[\mathrm{sec}^{-1}\right]$ \\
\hline LO $-\Lambda=500 \mathrm{MeV}$ & 238.8 & 381.7 & 1362 \\
LO $-\Lambda=600 \mathrm{MeV}$ & 238.7 & 380.8 & 1360 \\
TOT $-\Lambda=500 \mathrm{MeV}$ & $254.4 \pm 9$ & $399.2 \pm 9$ & $1488 \pm 9$ \\
TOT $-\Lambda=600 \mathrm{MeV}$ & $255 \pm 1$ & $399 \pm 1$ & $1499 \pm 9$ \\
\hline
\end{tabular}

Muon capture on $A=2,3$ nuclei. The muon capture reactions here under consideration are $\mu^{-}+d \rightarrow$ $n+n+\nu_{\mu}$ and $\mu^{-}+{ }^{3} \mathrm{He} \rightarrow{ }^{3} \mathrm{H}+\nu_{\mu}$, for which we are interested in the capture rate in the doublet hyperfine initial state $\left(\Gamma^{D}\right)$ and in the total capture rate $\left(\Gamma_{0}\right)$, respectively. The $\chi$ EFT results of Ref. [5] are summarized in Table 2, where the value for $\Gamma^{D}$ obtained retaining in the final $n n$ scattering state only the ${ }^{1} S_{0}$ partial wave is also shown. The results of the table can be summarized as $\Gamma^{D}=(399 \pm 3)$ $\sec ^{-1}$ and $\Gamma_{0}=(1494 \pm 21) \mathrm{sec}^{-1}$. The errors are due to cutoff dependence, the uncertainty inherent the fitting procedure of the LEC $d_{R}$, and radiative corrections [5]. The experimental data for $\Gamma^{D}$ are affected at present by quite large uncertainties and no significant comparison between theory and experiment can be made. Instead, the theoretical prediction for $\Gamma_{0}$ is in very nice agreement with the experimental determination of Ref. [15], $(1496 \pm 4) \mathrm{sec}^{-1}$.

Weak proton-proton capture. The astrophysical $S$-factor for $p p$ weak capture is typically given as a Taylor expansion around $E=0$, and the coefficients of the expansion, $S(0), S^{\prime}(0)$ and $S^{\prime \prime}(0)$, are the quantities of interest [16]. Alternatively, the energy dependence of $S(E)$ can be made explicit by calculating it directly. Note that the Gamow peak for the $p p$ reaction is at $E=6 \mathrm{keV}$ in the Sun, and it becomes of about $15 \mathrm{keV}$ in larger mass stars. Therefore, we have studied the $p p$ reaction in the energy range $E=3-100 \mathrm{keV}$. Two ingredients are essential in the calculation: (i) the initial $p p$ scattering state is calculated using the $\chi$ EFT N3LO potential [3] augmented not only of the Coulomb interaction, but also of the higher order electromagnetic terms, due to two-photon exchange and vacuum polarization. The additional distortion of the $p p$ wave function, induced primarily by vacuum polarization, has been shown to reduce $S(0)$ by $\sim 1 \%$ in previous studies (see Ref. [16] and references therein). (ii) To have the correct energy-dependence of the $S$-factor up to $E=100 \mathrm{keV}$, we have included, in addition to the $S$-wave (the ${ }^{1} S_{0}$ partial wave), all the $P$-wave channels $\left({ }^{3} P_{0},{ }^{3} P_{1}\right.$, and $\left.{ }^{3} P_{2}\right)$, and we have retained the explicit dependence on the momentum transfer $\mathbf{q}=\mathbf{p}_{e}+\mathbf{p}_{\nu}$ ( $\mathbf{p}_{e}$ and $\mathbf{p}_{\nu}$ are the electron and neutrino momenta, respectively) via a standard multipole expansion. More details can be found in Ref. [6]. Finally, we recall that the model for the weak current is the one discussed in Sec. 22 and is the same used in the successful studies of the muon capture reactions presented above. The $S$-factor at zero energy is found to be $S(0)=(4.030 \pm 0.006) \times 10^{-23} \mathrm{MeV} \mathrm{fm}^{2}$, with a $P$-wave contribution of $0.020 \times 10^{-23} \mathrm{MeV} \mathrm{fm}^{2}$. The theoretical uncertainty is due to the fitting procedure of the LECs and to the cutoff dependence. This value is $\simeq 1 \%$ larger than the value reported in the literature [16]. The energy dependence of $S(E)$ is shown in Fig. 3. The $S$ - and $(S+P)$-wave contributions are displayed separately, and the theoretical uncertainty is included - the curves are in fact very narrow bands. As expected, the $P$-wave contributions become significant at higher values of $E$. From these results, with a least $\chi^{2}$ fitting procedure, we have calculated the coefficients $S^{\prime}(0)$ and $S^{\prime \prime}(0)$, which are listed in Table II of Ref. [6], where a thorough discussion of these results is also present.

\section{Conclusions and outlook}

We have presented the most recent studies of EW observables involving light nuclei within the $\chi \mathrm{EFT}$ framework. The considered observables are in good agreement with the available experimental data, except for the $A=3$ magnetic form factors at high values of momentum transfer, in a region, though, well beyond the applicability range of $\chi \mathrm{EFT}$. To be noticed that, while the EM observables have been studied with the most recent model for the $\chi \mathrm{EFT}$ EM operators up to N3LO, the weak observables have been studied with the model of Park et al. up to N2LO. Therefore, it is highly desirable to derive 


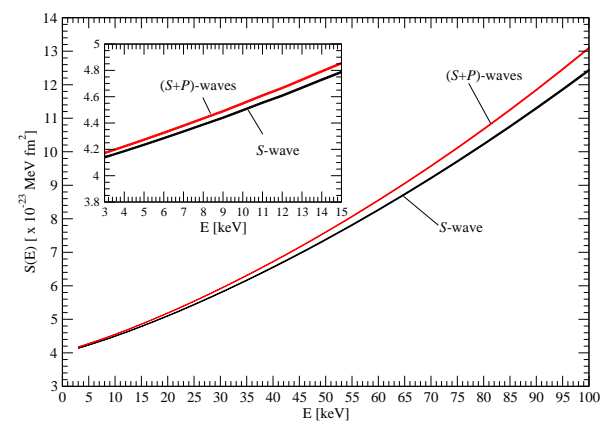

Fig. 3 The astrophysical $S$-factor for $E=3-100 \mathrm{keV}$. The $S$ - and $(S+P)$-wave contributions are displayed separately. In the inset, $S(E)$ is shown in the range $3-15 \mathrm{keV}$.

the axial currents up to N3LO in TOPT and to repeat the studies for the muon captures and $p p$ reaction. Finally, it should be noticed that the radiative proton-deuteron and the weak proton- ${ }^{3} \mathrm{He}$ captures, also relevant in astrophysics, are at reach within the present framework. Work along these lines is currently underway.

Acknowledgements The Author would like to thank the Gran Sasso Science Institute (INFN), and especially Prof. F. Vissani, for the support and warm hospitality extended to her in occasion of her visit in the Fall 2013 during which this work was completed.

\section{References}

1. Park T.S. et al. (1996) Chiral Lagrangian approach to exchange vector currents in nuclei. Nucl. Phys. A 596: $515-552$

2. Park T.S. et al. (2003) Parameter free effective field theory calculation for the solar proton fusion and hep processes. Phys. Rev. C 67: 055206

3. Entem D.R. and Machleidt R. (2003) Accurate charge-dependent nucleon-nucleon potential at fourth order of chiral perturbation theory. Phys. Rev. C 68: 041001

4. Navràtil P. (2007) Local three-nucleon interaction from chiral effective field theory. Few-Body Syst. 41: $117-140$

5. Marcucci L.E. et al. (2012) Chiral effective field theory predictions for muon capture on deuteron and ${ }^{3} \mathrm{He}$. Phys. Rev. Lett. 108: 052502

6. Marcucci L.E. et al. (2013) Proton-proton weak capture in chiral effective field theory. Phys. Rev. Lett. 110: 192503

7. Pastore S. et al. (2009) Electromagnetic currents and magnetic moments in chiral effective field theory ( $\chi$ EFT). Phys. Rev. C 80: 034004; Pastore S. et al. (2011) Two-nucleon electromagnetic charge operator in chiral effective field theory $(\chi \mathrm{EFT})$ up to one loop. Phys. Rev. C 84: 024001

8. Kölling S. et al. (2009) Two-pion exchange electromagnetic current in chiral effective field theory using the method of unitary transformation. Phys. Rev. C 80: 045502; Kölling S. et al. (2011) Two-nucleon electromagnetic current in chiral effective field theory: One-pion exchange and short-range contributions. Phys. Rev. C 84: 054008

9. Piarulli M. et al. (2013) Electromagnetic structure of $A=2$ and 3 nuclei in chiral effective field theory. Phys. Rev. C 87: 014006

10. Gårdestig A. and Phillips D.R. (2006) How Low-Energy Weak Reactions Can Constrain Three-Nucleon Forces and the Neutron-Neutron Scattering Length. Phys. Rev. Lett. 96: 232301

11. Viviani M. et al. (2013) Effect of three-nucleon interaction in $p-{ }^{3} \mathrm{He}$ elastic scattering. Few Body Syst. 54: 885-890; and Phys. Rev. Lett. 111: 172302

12. Wiringa R.B. et al. (1995) Accurate nucleon-nucleon potential with charge-independence breaking. Phys. Rev. C 51: 38-51

13. Pudliner B.S. et al. (1995) Quantum Monte Carlo Calculations of $A \leq 6$ Nuclei. Phys. Rev. Lett. 74: 4396-4399

14. Marcucci L.E. et al. (1998) Electromagnetic structure of trinucleons. Phys. Rev. C 58: 3069-3084; Marcucci L.E. et al. (2005) Electromagnetic structure of $A=2$ and 3 nuclei and the nuclear current operator. Phys. Rev. C 72: 014001

15. Ackerbauer P. et al. (1998) A precision measurement of nuclear muon capture on ${ }^{3}$ He. Phys. Lett. B 417: 224-232

16. Adelberger E.G. et al. (2011) Solar fusion cross sections. II. The $p p$ chain and CNO cycles. Rev. Mod. Phys. 83: 195-245 